\title{
Estresse ocupacional e fatores associados em servidores públicos de uma universidade federal do sul do Brasil
}

\author{
Occupational stress and associated factors among civil servants \\ of a federal university in the south of Brazil
}

Samuel Völz Lopes ${ }^{1}$

Marcelo Cozzensa da Silva ${ }^{1}$

${ }^{1}$ Programa de PósGraduação em Educação Física, Universidade Federal de Pelotas. R. Luís de Camões 625, Três Vendas. 96055-630 Pelotas RS Brasil.samuelvolzlopes@ gmail.com

\begin{abstract}
The aim of the study was to verify the prevalence of stress and associated factors in technical-administrative employees of a federal university of the south of Brazil. The sample was comprised of 371 civil servants. Sociodemograph$i c$, behavioral and occupational variables were collected. The occupational stress was measured by the Job Stress Scale (JSS). Data analysis was performed using descriptive and inferential statistics, through bivariate and multivariate analysis. Most subjects were female (57.4\%) and the average age and education of the sample were, respectively, 45.1 years and 18 years of study. The workplace infrastructure was classified as inadequate by $42 \%$ of subjects. Of the civil servants, $22.7 \%$ were classified as being in high stress positions and 28\% in passive work routines. In the adjusted analysis, occupational stress was associated with a lower level of education, poor workplace infrastructure and less social support. Individuals with adequate structure had odds of 2.79 and 2.30, respectively, of being in low stress and passive job positions. These aspects should be the focus of interventions by university managers to reduce the level of stress and risk of illness.
\end{abstract}

Key words Occupational stress, Motor activity, Occupational health, Professional Burnout, Lifestyle
Resumo $O$ objetivo do estudo foi verificar a prevalencia de estresse e os fatores associados em servidores técnico-administrativos de uma universidade federal do sul do Brasil. A amostra foi composta por 371 servidores públicos. Foram coletadas variáveis sociodemográficas, comportamentais e de trabalho. O estresse no trabalho foi mensurado pelo Job Stress Scale (JSS). Para a análise dos dados foram realizados cálculos de estatística descritiva e inferencial, através de análise bivariada e multivariável. A maioria dos indivíduos era do sexo feminino $(57,4 \%)$ e as médias de idade e escolaridade encontradas para a amostra foram, respectivamente, de 45,1 e de 18 anos de estudo. A estrutura de trabalho foi classificada como inadequada por $42 \%$ dos indivíduos. Entre os servidores, 22,7\% foram classificados em alta exigência e $28 \%$ em trabalho passivo. $\mathrm{Na}$ análise ajustada, o estresse no trabalho mostrou-se associado à menor escolaridade, pior estrutura de trabalho e menor apoio social. Individuos com estrutura adequada tiveram odds de 2,79 e 2,30, respectivamente, para baixa exigência e trabalho passivo. Estes aspectos devem ser foco de intervenção por parte dos gestores da universidade no sentido de reduzir o nível de estresse e risco de adoecimento.

Palavras-chave Estresse ocupacional, Atividade motora, Saúde do trabalhador, Esgotamento profissional, Estilo de vida 


\section{Introdução}

A saúde do trabalhador está em destaque no meio científico e é foco de pesquisas e debates internacionais. Um dos principais fatores relacionados ao trabalho que comprometem a saúde é o estresse laboral. Os impactos negativos causados pelo estresse afetam profissionais de diferentes áreas $^{1}$ e provocam preocupação entre os gestores devido ao crescente número de afastamentos por motivos de saúde ${ }^{2}$. Porém, situações envolvendo estresse estão presentes diariamente no ambiente de trabalho e são difíceis de serem evitadas.

O estresse faz parte da vida de todas as pessoas, porém, em níveis mais elevados pode provocar uma série de doenças e morbidades, nas quais estão a depressão, a ansiedade ${ }^{3,4}$, a exaustão emocional $^{3} \mathrm{e}$ as doenças cardíacas 5 . Dados do governo britânico (Health and Safety Executive) revelam que a prevalência de estresse entre os anos de 2011 e 2012 chegou a 40\% do total de doenças relatadas por trabalhadores no país 6 . No Brasil, as investigações científicas voltaram-se para as associações entre o estresse e a falta de ânimo ${ }^{7}$, os transtornos mentais comuns ${ }^{8,9} \mathrm{e}$ a hipertensão ${ }^{10}$.

Segundo Karasek ${ }^{11}$, o estresse laboral e o risco de adoecimento estão relacionados a dois aspectos: demandas e controle sobre o trabalho. Este trata da possibilidade do trabalhador utilizar seu intelecto e autoridade para a tomada de decisões sobre como realizar suas atividades. Já as demandas são pressões de natureza psicológica, que podem ser quantitativas, tais como tempo e velocidade na realização de tarefas, ou qualitativas, como os conflitos entre demandas contraditórias ${ }^{12}$.

As combinações dos níveis alto e baixo das dimensões de demanda e controle podem determinar o nível de estresse dos indivíduos. A alta exigência, que ocorre quando se tem alta demanda psicológica e baixo controle sobre o trabalho, gera maiores riscos à saúde, proporcionando fadiga, depressão e ansiedade ${ }^{13}$. O trabalho passivo pode acarretar perdas de habilidade e desinteresse por ter como característica o baixo controle sobre as funções laborais. O trabalho ativo, formado pela junção da alta demanda com o alto controle, é menos insalubre, à medida que os trabalhadores podem planejar suas ações de acordo com as suas capacidades $^{12}$. A baixa exigência é apresentada como a situação laboral ideal, pois comporta as baixas demandas com alto controle.

As atividades exercidas no trabalho por técnico-administrativo de universidades são em sua maioria atividades burocráticas, que demandam grandes responsabilidades e exigem alto nível de concentração. Tais atividades podem ocasionar tensões entre os funcionários, se os mesmos não se sentirem capazes, ou não obtiverem meios para realizá-las. Estudos apontam que o estresse laboral atinge grande parte dos servidores públicos, devido as altas responsabilidades embutidas nas decisões sobre processos administrativos, grande quantidade de atendimento ao público e, ainda, está relacionado com fatores físicos, sociais e emocionais ${ }^{14,15}$. No entanto, os servidores da região sul do Brasil ainda são pouco estudados em relação as causas do estresse e, assim, os dados do presente estudo contribuirão para o avanço do conhecimento da saúde do trabalhador.

O campo da saúde necessita de maiores investigações sobre aspectos laborais causadores de morbidades, por isso, objetivou-se investigar a prevalência de estresse e sua relação com variáveis sociodemográficas, comportamentais e de características de trabalho em técnico-administrativos de uma universidade pública do sul do Brasil.

\section{Métodos}

Trata-se de um estudo observacional de caráter transversal em uma amostra dos servidores técnico-administrativos da Universidade Federal de Pelotas (UFPel), nos anos de 2014/2015. A mesma universidade está situada, em seus campus principais, nos municípios de Pelotas e Capão do Leão, ambos distantes a, aproximadamente, 250 quilômetros ao sul de Porto Alegre, capital do estado do Rio Grande do Sul. A UFPel contava até a data de 22/05/2014 com um efetivo de 1356 servidores técnico-administrativos.

Foi realizado um cálculo de tamanho amostral para estimar a prevalência de estresse e outro para estudo de associação entre ele e as variáveis independentes em estudo. Estimando-se uma prevalência de $54,0 \%$ de ativos no lazer, um erro absoluto de 5,0\% e um nível de significância de $95 \%$, estimou-se, dentre todos os cálculos, que a maior amostra necessária seria de 382 indivíduos. A este valor foram acrescidos (20\%) 77 sujeitos referentes a perdas e recusas, totalizando 459 entrevistas.

Quanto aos aspectos logísticos, inicialmente foi solicitada a autorização do estudo a vice-reitora da universidade e realizada uma pactuação com a Pró-Reitoria de Gestão de Pessoas da UFPel para delinear a melhor forma de acesso aos servidores. A partir daí, buscou-se a lista de 
todos os funcionários técnico-administrativos da universidade e das unidades de trabalho na qual estavam alocados.

O processo de amostragem foi realizado em múltiplos estágios. Primeiramente, todas unidades acadêmicas e administrativas foram agrupadas por áreas afins, sendo elas: reitoria e unidades administrativas, ciências exatas, humanas e biológicas. As áreas, então, foram ordenadas, de maior a menor, segundo o número de técnico -administrativos. Conhecido o número de pessoas a serem amostradas, foi estabelecido um pulo sistemático de três indivíduos, a começar por um indivíduo sorteado da maior área de agrupamento, até atingir-se o número estimado. Todos indivíduos sorteados para a amostra foram procurados em suas unidades de trabalho e informados dos objetivos, riscos e contribuições do estudo e, então, convidados a participar, mediante à apresentação e solicitação da assinatura do Termo de Consentimento Livre e Esclarecido (TCLE), para posterior preenchimento do questionário. Com os indivíduos não encontrados na primeira tentativa, foram realizadas, pelo menos, outras três tentativas de contato no local de trabalho, e, se ainda não fossem encontrados, buscou-se o contato telefônico ou via e-mail, junto ao setor de Gestão de Pessoas ou colegas de trabalho. É importante ressaltar que o espaçamento de tempo entre as tentativas foi adaptado de acordo com o motivo do desencontro. Foram excluídos os servidores que estavam afastados para a realização de cursos de capacitação (especialização, mestrado, doutorado) fora da cidade ou algum outro tipo de afastamento.

Foi realizado um estudo piloto em julho de 2014, com dez técnico-administrativos da reitoria do Instituto Federal de Educação, Ciência e Tecnologia Sul-Rio-Grandense, para testar o entendimento das questões, monitorar o tempo de aplicação, servir como treinamento para o pesquisador e aperfeiçoar o instrumento para sua versão final.

A coleta de dados ocorreu no período de julho de 2014 a janeiro de 2015, por entrevistadores previamente treinados. $\mathrm{O}$ instrumento utilizado foi um questionário de aplicação autopreenchido, contendo informações sociodemográficas (idade, sexo, cor da pele, situação conjugal, escolaridade, peso e altura para o cálculo do Índice de Massa Corporal - IMC), comportamentais (nível de atividade física, horas de sono, uso de medicamentos, tabagismo e alcoolismo), de trabalho (horas trabalhadas na semana, acidentes de trabalho, tempo de serviço e conhecimento sobre a função) e de estresse laboral , segundo o Modelo Demanda-Controle (MDC).

Para a mensuração do nível de atividade física foi utilizado o International Physical Activity Questionnaire (IPAQ) versão longa, validado no Brasil por Matsudo et al. ${ }^{16}$. O mesmo foi mensurado nos domínios do lazer e deslocamento do IPAQ longo. Esses dois domínios são os mais relevantes para os níveis populacionais e para orientar políticas de saúde pública ${ }^{17}$. Foi avaliado o tempo total semanal despendido em atividades físicas de lazer e deslocamento para classificar os indivíduos como ativos, e o ponto de corte foi de 150 minutos de atividade física semanal ${ }^{18}$.

A variável nutricional foi definida através do cálculo do Índice de Massa Corporal (IMC) obtido pela divisão do peso (em quilogramas) pelo quadrado da altura (em metros). Para o cálculo, utilizou-se peso e altura auto-referidos e pontos de corte que classificaram os indivíduos nas seguintes categorias: baixo peso $(<18,5)$, eutrófico $(18,5$ a 24,9$)$, sobrepeso $(25$ a 29,9$)$ e obesidade $(\geq 30)^{19}$.

O desfecho em estudo, estresse no trabalho, foi mensurado utilizando-se a versão reduzida da Job Stress Scale (JSS), de Töres Theorell (1988), traduzida e validada no Brasil por Alves et al. ${ }^{12} \mathrm{e}$ Hökerberg et al. ${ }^{20}$. O instrumento contém cinco questões para avaliar a demanda psicológica no trabalho, seis para avaliar o controle sobre o trabalho e outras seis para avaliar o suporte social. Cada questão contém uma escala Likert (1 - 4), que vai de "nunca/nunca" a "frequentemente", com a qual se pode gerar um escore final ${ }^{11,12}$. O escore varia de 5-20 para a demanda psicológica e de 6-24 para controle e suporte social.

A definição dos quadrantes de demanda-controle foi feita de acordo com a análise das dimensões psicológicas e controle sobre o trabalho. As somas dos escores foram classificadas como "baixo" ou "alto" a partir da mediana ${ }^{12}$. A baixa demanda psicológica ( $\leq 12$ pontos), ou alta demanda ( $\geq 13$ pontos), foi definida a partir da mediana de 12 ( \pm 3$)$. O baixo controle ( $\leq 17$ pontos), ou alto controle ( $\geq 18$ pontos), foi definido a partir da mediana de $17( \pm 2,6)$. E o apoio social baixo ( $\leq 21$ pontos), ou alto ( $\geq 22$ pontos), foi definido a partir da mediana de $21( \pm 2,6)$.

Baseado nas dimensões demanda e controle (baixo e alto) do MDC, os quadrantes de demanda-controle foram categorizados em "alta exigência” (alta demanda psicológica e baixo controle); "trabalho ativo" (alta demanda psicológica e alto controle); "trabalho passivo" (baixa demanda psicológica e baixo controle) e "baixa exigência" 
(baixa demanda psicológica e alto controle) $)^{11,12}$. Ainda, foi verificada a estrutura adequada de trabalho, mensurada de forma dicotômica (adequada / inadequada).

Para estruturação do banco de dados foi utilizado programa EpiData 3.1, no qual foi realizado o processo de dupla digitação e, posteriormente, verificação de possíveis inconsistências entre os bancos. A análise de dados consistiu-se em três etapas. Primeiramente, foi realizada a análise descritiva dos dados (cálculo de médias e desvio-padrão para as variáveis contínuas e cálculo de proporções e IC para as variáveis categóricas). Em um segundo momento, foi conduzido a análise de associação bivariada entre as variáveis independentes e o desfecho estudado utilizandose o teste de Qui-quadrado de Pearson, sendo a associação estatística aferida para valor $\mathrm{p}<0,05$. Por último, para avaliar a associação do desfecho nominal em quatro categorias (MDC) com as variáveis independentes conjuntamente, foi utilizada a regressão logística multinomial com o auxílio do programa estatístico STATA 13.0. Esse tipo de análise permitiu estimar, na forma de Odds Ratio (OR), a associação de cada variável independente com a ocorrência de MDC, sendo utilizada a "alta exigência" como categoria de referência. Os OR para MDC puderam ser estimados simultaneamente, evitando-se o uso de múltiplos testes estatísticos e produzindo estimativas proporcionais entre si, comparáveis diretamente e com categoria de referência comum. A entrada das variáveis na análise respeitou a hierarquia de determinação do desfecho.

O modelo proposto para a hierarquia citada foi constituído de três níveis: o primeiro, onde estavam inseridas as variáveis demográficas (sexo, idade e cor da pele), o segundo em que estão as variáveis socioeconômicas (situação conjugal e escolaridade), e o terceiro que abrange as variáveis comportamentais (prática de atividade física, tabagismo, problemas relacionados ao uso de álcool, horas de sono, uso de medicamentos), emocional (estado emocional e depressão), estrutura de trabalho (estrutura adequada) e a variável nutricional (IMC) e, por último, as variáveis de estresse (baixa demanda, trabalho ativo, trabalho passivo e alta demanda).

Os efeitos das variáveis do primeiro nível foram controlados entre si; as do segundo nível foram controlados entre elas e para as do primeiro nível; as do terceiro nível foram controladas entre elas e para as dos dois níveis anteriores. Entraram no modelo hierarquizado de análise todas as variáveis que apresentaram, na análise bivariada, valor $\mathrm{p} \leq 0,2$. As variáveis que, na análise multivariada, também apresentaram valor $\mathrm{p} \leq 0,2$ permaneceram no modelo sempre que preenchiam os critérios para prováveis fatores de confusão. Para seleção das variáveis que permaneceram no modelo de regressão logística multinomial foi utilizado o processo de seleção para trás, ficando no modelo final todas variáveis que apresentaram valor $\mathrm{p}<0,05$.

O projeto de pesquisa foi aprovado pelo Comitê de Ética e Pesquisa (CEP) da Escola Superior de Educação Física da UFPel. O estudo também está de acordo com a Resolução $\mathrm{n}^{\circ} 466 / 12$, do Conselho Nacional de Saúde, cumprindo todos os deveres éticos que cabem à comunidade científica, e assegurando os direitos aos sujeitos da pesquisa.

\section{Resultados}

Do total de servidores sorteados, 371 consentiram em participar do estudo e responderam o questionário. Foi considerado perda o total de $15,5 \%$ e ainda, $3,7 \%$ se recusaram a participar. As perdas e recusas foram coerentes com a distribuição de sexo e idade dos amostrados. A maioria dos entrevistados era do sexo feminino $(57,4 \%)$, com média de idade de 45,1 anos ( $\mathrm{DP}=11,7)$, casado ou com união estável $(66,0 \%)$ e de cor da pele branca $(83,6 \%)$. A média de escolaridade foi de 18 anos de estudo ( $D P=6,2)$, sendo que quase $50,0 \%$ dos entrevistados possuía 18 anos ou mais de estudo. Em relação às variáveis comportamentais e nutricional (IMC), 61,1\% dos entrevistados eram ativos fisicamente, $27,6 \%$ relataram já ter fumado, $13,5 \%$ eram fumantes e $63,8 \%$ dos entrevistados estavam nas categorias referentes a sobrepeso/obesidade (Tabela 1). A média de horas de sono registrada foi de 6,9 horas ( $\mathrm{DP}=1,8)$ de segunda a quinta e de 8,1 horas $(\mathrm{DP}=2,6)$ de sexta a domingo.

Em relação às características laborais, os servidores estavam alocados, predominantemente, nas unidades biológicas $(35,0 \%)$ seguido dos trabalhadores da reitoria $(24,1 \%)$. A média declarada da carga horária de trabalho semanal foi de 35,5 horas $(\mathrm{DP}=12,6)$ e o tempo médio de serviço como servidor da UFPel foi de 14,4 anos $(\mathrm{DP}=11,4)$. Com referência ao relato sobre a estrutura de trabalho, $42,0 \%$ dos indivíduos consideraram a mesma como inadequada.

A respeito dos cargos, $16,6 \%(n=71)$ dos entrevistados eram assistentes em administração, $10,2 \%(n=37)$ auxiliares de enfermagem, 6,1\% 
Tabela 1. Descrição da amostra segundo variáveis sociodemográficas e comportamentais dos técnicoadministrativos da UFPel, 2014.

\begin{tabular}{|c|c|c|}
\hline Variáveis & $\mathbf{N}$ & $\%$ \\
\hline \multicolumn{3}{|l|}{ Sexo } \\
\hline Masculino & 158 & 42,6 \\
\hline Feminino & 213 & 57,4 \\
\hline \multicolumn{3}{|l|}{ Idade } \\
\hline $20 / 29$ & 42 & 11,3 \\
\hline $30 / 39$ & 88 & 23,7 \\
\hline $40 / 49$ & 85 & 22,9 \\
\hline $50 / 59$ & 115 & 31 \\
\hline 60 ou mais & 41 & 11,1 \\
\hline \multicolumn{3}{|l|}{ Situação Conjugal } \\
\hline Casado / união estável & 245 & 66 \\
\hline Solteiro & 76 & 20,5 \\
\hline Separado & 39 & 10,5 \\
\hline Viúvo & 11 & 3 \\
\hline \multicolumn{3}{|l|}{ Cor da Pele } \\
\hline Branca & 310 & 83,6 \\
\hline Negra & 31 & 8,4 \\
\hline Parda/Mulata & 26 & 7 \\
\hline Outra & 4 & 1 \\
\hline \multicolumn{3}{|l|}{ Escolaridade } \\
\hline $0 / 12$ anos & 52 & 15,3 \\
\hline $13 / 17$ anos & 119 & 34,9 \\
\hline 18 ou mais anos & 170 & 49,8 \\
\hline \multicolumn{3}{|l|}{ IMC } \\
\hline Baixo peso & 4 & 1,1 \\
\hline Eutrófico & 127 & 35,1 \\
\hline Sobrepeso & 150 & 41,4 \\
\hline Obesidade & 81 & 22,4 \\
\hline \multicolumn{3}{|l|}{ Fumo } \\
\hline Nunca fumou & 218 & 58,9 \\
\hline Fumante & 50 & 13,5 \\
\hline Ex-fumante & 102 & 27,6 \\
\hline \multicolumn{3}{|l|}{ Atividade Física Total $\dagger$} \\
\hline Insuficientemente ativos & 119 & 38,9 \\
\hline Ativos & 187 & 61,1 \\
\hline
\end{tabular}

( $\mathrm{n}=22)$ médicos, 4,7\% $(\mathrm{n}=17)$ enfermeiros e $4,7 \%(\mathrm{n}=17)$ técnicos de laboratório. Ainda, foram indicados outros 96 cargos que correspondem ao restante $(57,7 \%)$ dos entrevistados.

A classificação dos técnico-administrativos segundo os quadrantes do MDC foi: $22,7 \%$ apresentarem alta exigência, 22,4\% trabalho ativo, $28 \%$ trabalho passivo e $26,9 \%$ baixa exigência.

Dos 306 indivíduos que responderam todas as questões sobre atividade física, $187(61,1 \%)$ atingiram a recomendação de pelo menos 150 minutos de atividade física semanal nos domínios do lazer e deslocamento. Quando analisado apenas o domínio do lazer, 317 sujeitos responderam o questionário e, destes, 43,2\% eram fisicamente ativos.

No que se refere às variáveis sociodemográficas e aos quadrantes do MDC, a única variável que demonstrou estar associada com os grupos de demanda-controle foi a escolaridade (Tabela 2). Para esta variável, os testes indicaram que $58,7 \%$ dos indivíduos com até 12 anos de estudo possuem um trabalho passivo, ou seja, baixa demanda e baixo controle sobre o trabalho.

Quando foram testados os hábitos comportamentais como horas de sono, uso de medicação para estresse e ansiedade, fumo, prática de atividade física e problemas relacionados ao álcool, nenhuma das variáveis esteve associada com os quadrantes do MDC nos técnicos-administrativos da UFPel (Tabela 3).

As características laborais segundo os quadrantes do modelo MDC mostraram diferenças significativas entre os grupos avaliados para as variáveis de horas semanais trabalhadas ( $p$ $=0,002)$, estrutura de trabalho adequada ( $\mathrm{p}<$ $0,001)$ e apoio social ( $\mathrm{p}=0,002)$, conforme mostra a Tabela 4. A maior parte dos indivíduos que trabalhavam 36 ou mais horas por semana apresentou um trabalho ativo (alta demanda e alto controle). Quando a estrutura de trabalho foi referida como adequada, a maioria dos indivíduos teve escores relativos à baixa exigência segundo o MDC. O quadrante baixa exigência também esteve associado à maioria dos sujeitos $(35,2 \%)$ que relataram alto apoio social.

$\mathrm{Na}$ análise multinomial, à medida que aumentou o nível educacional dos avaliados, maior foi o quadro de baixa exigência $(p=0,03)$ e trabalho ativo $(\mathrm{p}<0,001)$. Com relação à estrutura adequada de trabalho, aqueles que afirmaram ter estrutura adequada tiveram odds de 2,8 e 2,3, respectivamente, para baixa exigência e trabalho passivo, quando comparados ao grupo de referência. Também, indivíduos com alto apoio social, apresentaram maior chance de baixa exigência.

\section{Discussão}

Em relação aos dados demográficos, podemos observar homogeneidade com outros estudos com populações de servidores ${ }^{2,8,21}$ nas variáveis como sexo, idade, cor da pele, escolaridade e situação conjugal. Entretanto, esses estudos não 
Tabela 2. Associação entre variáveis sociodemográficas e os quadrantes demanda-controle da JSS, dos servidores técnico-administrativos da UFPel, 2014.

\begin{tabular}{|c|c|c|c|c|c|c|c|c|c|}
\hline \multirow{3}{*}{ Variáveis Sociodemográficas } & \multicolumn{9}{|c|}{ Quadrantes demanda-controle } \\
\hline & \multicolumn{2}{|c|}{ Baixa Exigência } & \multicolumn{2}{|c|}{ Trabalho Passivo } & \multicolumn{2}{|c|}{ Trabalho Ativo } & \multicolumn{2}{|c|}{ Alta Exigência } & \multirow{2}{*}{$\mathbf{p}^{*}$} \\
\hline & $\mathbf{n}$ & $\%$ & $\mathbf{n}$ & $\%$ & $\mathbf{n}$ & $\%$ & $\mathbf{n}$ & $\%$ & \\
\hline Sexo (353) & & & & & & & & & 0,659 \\
\hline Masculino & 38 & 24,8 & 48 & 31,4 & 33 & 21,6 & 34 & 22,2 & \\
\hline Feminino & 57 & 28,5 & 51 & 25,5 & 46 & 23 & 46 & 23 & \\
\hline Idade (353) & & & & & & & & & 0,131 \\
\hline $20 / 29$ & 18 & 43,9 & 11 & 26,8 & 5 & 12,2 & 7 & 17,1 & \\
\hline $30 / 39$ & 23 & 26,4 & 20 & 23 & 21 & 24,1 & 23 & 26,4 & \\
\hline $40 / 49$ & 19 & 23,7 & 21 & 25,9 & 25 & 30,9 & 16 & 19,8 & \\
\hline $50 / 59$ & 27 & 24,6 & 32 & 29,1 & 22 & 20 & 29 & 26,4 & \\
\hline 60 ou mais & 8 & 23,5 & 15 & 44,1 & 6 & 17,7 & 5 & 14,7 & \\
\hline Escolaridade (324) & & & & & & & & & $<0,001$ \\
\hline 0/12 anos & 6 & 13,0 & 27 & 58,7 & 4 & 8,7 & 9 & 19,6 & \\
\hline $13 / 17$ anos & 32 & 29,1 & 31 & 28,2 & 15 & 13,6 & 32 & 29,1 & \\
\hline 18 ou mais anos & 51 & 30,4 & 34 & 20,2 & 55 & 32,7 & 28 & 16,7 & \\
\hline Cor da Pele (353) & & & & & & & & & 0,117 \\
\hline Brancos & 87 & 29,4 & 80 & 27,0 & 65 & 22 & 64 & 21,6 & \\
\hline Outra & 8 & 14,0 & 19 & 33,3 & 14 & 24,6 & 16 & 28,1 & \\
\hline Situação Conjugal (353) & & & & & & & & & 0,829 \\
\hline Casado / com companheiro & 64 & 27,4 & 62 & 26,5 & 53 & 22,7 & 55 & 23,5 & \\
\hline Solteiro / sem companheiro & 31 & 26,1 & 37 & 31,1 & 26 & 21,9 & 25 & 21,0 & \\
\hline \multicolumn{10}{|l|}{ IMC (346) } \\
\hline Baixo peso & 1 & 25 & 1 & 25 & 2 & 50 & - & - & 0,785 \\
\hline Eutrófico & 34 & 28,1 & 30 & 24,8 & 31 & 25,6 & 26 & 21,5 & \\
\hline Sobrepeso & 39 & 26,7 & 47 & 32,2 & 29 & 19,9 & 31 & 21,2 & \\
\hline Obesidade & 19 & 25,3 & 20 & 26,7 & 16 & 21,3 & 20 & 26,7 & \\
\hline
\end{tabular}

*Teste Qui-quadrado de Pearson.

avaliaram o estado nutricional que, no presente trabalho, destacou-se pela elevada proporção $(63,4 \%)$ de indivíduos com sobrepeso/obesidade. A média das capitais brasileiras é de 52,5\% de adultos com excesso de peso ${ }^{22}$. Em Pelotas, foi conduzido um estudo, no ano de 2010, indicando que $62,4 \%$ dos sujeitos estavam nas categorias de sobrepeso e obesidade do cálculo do IMC ${ }^{23}$. $\mathrm{O}$ mesmo estudo apontou que alguns fatores podem contribuir para o aumento da obesidade como variáveis socioeconômicas, sociopolíticas, escolaridade ${ }^{23}$. Outros fatores relacionados ao sobrepeso e obesidade devem ser observados, como as longas jornadas de trabalho exercidas de maneira sentada, caracterizando comportamento sedentário ${ }^{24}$. Esse tipo de atividade é característica da função laboral da maioria dos técnicos-administrativos da UFPel.

Através do IPAQ, encontramos que $61,1 \%$ dos indivíduos eram fisicamente ativos. Outros estudos conduzidos no Brasil e que também uti- lizaram o IPAQ com população de servidores de universidades públicas, mostraram que, na Universidade Estadual da Bahia, 50,6\% dos sujeitos eram ativos fisicamente ${ }^{25}$ e na Universidade Estadual do Piauí, 53,6\% dos funcionários dos setores administrativos eram moderadamente ativos e $13,9 \%$ eram muito ativos ${ }^{24}$. Porém, não foi possível realizar maiores comparações devido a estes estudos utilizarem a versão curta do IPAQ, a qual não mensura os níveis de atividade física nos mesmos moldes da versão longa.

A formação dos quadrantes do MDC indicada de forma quantitativa por um ponto de corte à partir da mediana da demanda psicológica e do controle sobre o trabalho, mostrou valores aproximados em todas as categorias, mas com maiores percentuais na categoria de trabalho passivo. Os achados corroboram com alguns estudos nacionais, com populações de enfermeiros ${ }^{13} \mathrm{e}$ técnico-administrativos ${ }^{10}$, com prevalências de $35,6 \%$ e $28,3 \%$ de trabalho passivo, respectiva- 
Tabela 3. Associação entre variáveis comportamentais e os quadrantes demanda-controle da JSS, dos servidores técnico-administrativos da UFPel, 2014.

\begin{tabular}{|c|c|c|c|c|c|c|c|c|c|}
\hline \multirow{3}{*}{ Variáveis Comportamentais } & \multicolumn{9}{|c|}{ Quadrantes demanda-controle } \\
\hline & \multicolumn{2}{|c|}{$\begin{array}{c}\text { Baixa } \\
\text { Exigência }\end{array}$} & \multicolumn{2}{|c|}{$\begin{array}{c}\text { Trabalho } \\
\text { Passivo }\end{array}$} & \multicolumn{2}{|c|}{$\begin{array}{c}\text { Trabalho } \\
\text { Ativo }\end{array}$} & \multicolumn{2}{|c|}{$\begin{array}{c}\text { Alta } \\
\text { Exigência }\end{array}$} & \multirow{2}{*}{$\mathbf{p}^{*}$} \\
\hline & n & $\%$ & $\mathbf{N}$ & $\%$ & $\mathrm{n}$ & $\%$ & n & $\%$ & \\
\hline Problemas relacionados ao álcool (326) & & & & & & & & & 0,457 \\
\hline Não & 90 & 28,9 & 86 & 27,7 & 66 & 21,2 & 69 & 22,2 & \\
\hline Sim & 4 & 26,7 & 6 & 40 & 4 & 26,7 & 1 & 6,7 & \\
\hline Atividade física total $\dagger(292)$ & & & & & & & & & 0,097 \\
\hline Insuficientemente ativos & 24 & 21,4 & 34 & 30,4 & 24 & 21,4 & 30 & 26,8 & \\
\hline Suficientemente ativos & 58 & 32,2 & 54 & 30 & 38 & 21,1 & 30 & 16,7 & \\
\hline Atividade física de lazer $†$ (302) & & & & & & & & & 0,052 \\
\hline Insuficientemente ativos & 44 & 25,7 & 53 & 31 & 31 & 18,1 & 43 & 25,2 & \\
\hline Ativos & 41 & 31,3 & 36 & 27,5 & 35 & 26,7 & 19 & 14,5 & \\
\hline Horas de sono semanais ( 350 ) & & & & & & & & & 0,176 \\
\hline Até 6h & 35 & 25,7 & 32 & 23,5 & 38 & 27,9 & 31 & 22,8 & \\
\hline 7h ou mais & 60 & 28 & 66 & 30,8 & 40 & 18,7 & 48 & 22,4 & \\
\hline Horas de sono no final de semana (368) & & & & & & & & & 0,261 \\
\hline Até $6 \mathrm{~h}$ & 16 & 23,2 & 15 & 21,7 & 17 & 24,6 & 21 & 30,4 & \\
\hline 7h ou mais & 78 & 27,8 & 83 & 29,5 & 61 & 21,7 & 59 & 21 & \\
\hline Fumo (352) & & & & & & & & & 0,544 \\
\hline Nunca fumou & 59 & 28,4 & 52 & 25 & 49 & 23,6 & 48 & 23,1 & \\
\hline Fumante ou ex & 36 & 25 & 46 & 31,9 & 30 & 20,8 & 32 & 22,2 & \\
\hline Uso de medicação (351) & & & & & & & & & 0,542 \\
\hline Não & 81 & 27,6 & 83 & 28,2 & 68 & 23,1 & 62 & 21,1 & \\
\hline Sim & 14 & 24,7 & 15 & 26,3 & 11 & 19,3 & 17 & 29,8 & \\
\hline
\end{tabular}

* Teste Qui-quadrado de Pearson. Ponto de corte de 150 minutos semanais.

mente. Porém outros estudos mostraram prevalências superiores em outros quadrantes de baixa exigência $(30,2 \%)^{21}$ e trabalho ativo $(43,2 \%)^{2}$. O que caracteriza o quadrante trabalho passivo é a baixa demanda e o baixo controle sobre o trabalho, que faz com que os indivíduos se tornem mais suscetíveis a desenvolver estresse laboral e a apresentar perda de habilidade e desinteresse ${ }^{12}$.

As variáveis que estiveram associadas significativamente com os quadrantes do MDC foram a escolaridade, carga horária semanal, estrutura de trabalho e apoio social. Em relação a escolaridade, a análise mostrou que os indivíduos que possuem menor grau escolar apresentam baixo controle sobre o trabalho. Outros estudos ${ }^{9,26}$ mostraram que os profissionais de enfermagem com menor grau de instrução, principalmente os não graduados, estiveram associados ao trabalho passivo e alto desgaste. De acordo com Mark e Smith ${ }^{27}$, o estresse no trabalho está negativamente associado às habilidades para desempenhar tarefas. Porém, não se descarta o viés de que cargos de maior escolarização exigem maior controle sobre o trabalho.

Há escassez na literatura de estudos de associação entre estresse e atividade física. Alguns mostram evidências de menores índices de estresse nos indivíduos que praticam atividades físicas $^{28-30}$. Mas esse fato não é consenso. Por exemplo, estudo de revisão da World Health Organization $^{30}$ incluía pesquisas que não encontraram associação entre essas duas variáveis. Mais recentemente, um estudo conduzido no Brasil por Greco et al. ${ }^{21}$ testou a associação do MDC com a prática de atividade física e não encontrou resultados estatisticamente significativos. Nos servidores técnicos-administrativos da UFPel, os níveis de estresse laboral, segundo o MDC, também não foram significativamente associados à atividade física. Em uma segunda análise, testou-se apenas o domínio das atividades físicas de lazer com o MDC, porém, sem associação. Cabe ressaltar que em todas as pesquisas apresentadas houve variação de instrumentos para avaliar es- 
Tabela 4. Associação entre variáveis relacionadas ao trabalho e os quadrantes demanda-controle da JSS, dos servidores técnico-administrativos da UFPel, 2014.

\begin{tabular}{|c|c|c|c|c|c|c|c|c|c|}
\hline \multirow{3}{*}{$\begin{array}{c}\text { Variáveis Relacionadas } \\
\text { ao Trabalho }\end{array}$} & \multicolumn{9}{|c|}{ Quadrantes demanda-controle } \\
\hline & \multicolumn{2}{|c|}{$\begin{array}{c}\text { Baixa } \\
\text { Exigência }\end{array}$} & \multicolumn{2}{|c|}{$\begin{array}{c}\text { Trabalho } \\
\text { Passivo }\end{array}$} & \multicolumn{2}{|c|}{$\begin{array}{c}\text { Trabalho } \\
\text { Ativo }\end{array}$} & \multicolumn{2}{|c|}{$\begin{array}{c}\text { Alta } \\
\text { Exigência }\end{array}$} & \multirow[t]{2}{*}{$\mathbf{p}^{*}$} \\
\hline & $\mathbf{n}$ & $\%$ & $\mathbf{n}$ & $\%$ & $\mathbf{n}$ & $\%$ & $\mathbf{n}$ & $\%$ & \\
\hline Horas semanais trabalhadas (350) & & & & & & & & & 0,002 \\
\hline$\leq 35 \mathrm{~h}$ & 53 & 29,9 & 58 & 32,8 & 25 & 14,1 & 41 & 23,2 & \\
\hline$\geq 36 \mathrm{~h}$ & 41 & 23,7 & 41 & 23,7 & 53 & 30,9 & 38 & 22 & \\
\hline Conhecimento sobre a função (349) & & & & & & & & & 0,368 \\
\hline Parcial & 3 & 18,8 & 5 & 31,3 & 2 & 12,5 & 6 & 37,5 & \\
\hline Total & 92 & 27,6 & 94 & 28,2 & 77 & 23,1 & 70 & 21 & \\
\hline Acidente de trabalho (352) & & & & & & & & & 0,166 \\
\hline Não & 76 & 25,8 & 89 & 30,2 & 65 & 22 & 65 & 22 & \\
\hline Sim & 19 & 33,3 & 9 & 15,8 & 14 & 24,6 & 15 & 26,3 & \\
\hline Estrutura de trabalho adequada (351) & & & & & & & & & $<0,001$ \\
\hline Não & 29 & 19,2 & 35 & 23,2 & 42 & 27,8 & 45 & 29,8 & \\
\hline Sim & 66 & 33 & 63 & 31,5 & 37 & 18,5 & 34 & 17 & \\
\hline Tempo como servidor da UFPel (353) & & & & & & & & & 0,313 \\
\hline$\leq 15$ anos & 54 & 28,3 & 46 & 24,1 & 47 & 24,6 & 44 & 23,0 & \\
\hline$\geq 16$ anos & 41 & 25,3 & 53 & 32,7 & 32 & 19,8 & 36 & 22,2 & \\
\hline Apoio Social (348) & & & & & & & & & 0,002 \\
\hline Baixo & 38 & 20,4 & 48 & 25,8 & 48 & 25,8 & 52 & 28 & \\
\hline Alto & 57 & 35,2 & 49 & 30,3 & 29 & 17,9 & 27 & 16,7 & \\
\hline
\end{tabular}

* Teste Qui-quadrado de Pearson.

tresse e atividade física e isso pode ter ocasionado divergência entre os resultados.

A carga de trabalho elevada está fortemente associada com o estresse ocupacional ${ }^{4,27}$. As associações da carga horária com os quadrantes do MDC mostram maior frequência $(32,8 \%)$ de indivíduos com carga horária inferior à 36 horas semanais, que se enquadravam como trabalho passivo e com carga horária superior, enquadrados como trabalho ativo (30,9\%). O trabalho passivo é nocivo à saúde do trabalhador por não ter o controle adequado sobre o trabalho. No entanto, quando o trabalho ocorre de forma ativa, ainda que as demandas sejam excessivas, elas são menos danosas, porque, segundo Alves et al. ${ }^{12}$, o trabalhador possui meios para lidar com as dificuldades.

Para minimizar os danos à saúde, gerados pelas intensas e longas jornadas de trabalho, a Organização Internacional do Trabalho apontou medidas práticas de enfoque preventivo, tais como: ajustar a carga horária total, prevenir exigências excessivas por trabalhador, planejar prazos exequíveis, definir claramente as responsabilidades e evitar a subutilização das capacidades dos funcionários ${ }^{1}$. Concomitantemente, o perío- do de lazer também pode ser primordial na promoção da saúde e redução do estresse ocupacional, desde que englobe atividades de socialização, prática regular de atividade física e alimentação saudável. Tais atividades promovem o bem-estar psicológico e biológico dos indivíduos ${ }^{31}$.

Também em relação à carga de trabalho, foi conduzido um estudo na Suécia, o qual mostrou que os homens foram mais propensos a ter baixo nível de atividade física de lazer se expostos a horas extras de trabalho e aos quadrantes de trabalho passivo e alta exigência ${ }^{32}$. Contudo, não encontramos associação significativa da carga de trabalho com a prática de atividade física.

Quando a estrutura de trabalho foi referida como adequada, maioria dos indivíduos tiveram escores relativos à baixa exigência. Os achados do presente estudo, são semelhantes aos descritos na literatura internacional, que também mencionam a estrutura de trabalho associada negativamente com a percepção de estresse ${ }^{33,34}$. Lautizi et al. ${ }^{33}$ concluíram que a estrutura influencia diretamente na satisfação com o trabalho, ou seja, é correto afirmar que a estrutura adequada é fundamental para desenvolver um bom trabalho, sem ter que adaptar ou deixar de realizar ativi- 
Tabela 5. Análise logística multinomial para verificação da associação dos quadrantes demanda-controle da JSS, com variáveis independentes, dos servidores técnico-administrativos da UFPel, 2014.

\begin{tabular}{|c|c|c|c|c|c|c|c|c|c|}
\hline \multirow{3}{*}{$\begin{array}{c}\text { Variáveis } \\
\text { Relacionadas }\end{array}$} & \multicolumn{9}{|c|}{ Quadrantes demanda-controle } \\
\hline & \multicolumn{3}{|c|}{ Baixa Exigência } & \multicolumn{3}{|c|}{ Trabalho Passivo } & \multicolumn{3}{|c|}{ Trabalho Ativo } \\
\hline & OR & (IC95\%) & $\mathbf{p}$ & OR & (IC95\%) & $\mathbf{p}$ & OR & (IC95\%) & $\mathbf{p}$ \\
\hline Escolaridade & & & $0,03^{*}$ & & & 0,09 & & & $<0,001^{\star}$ \\
\hline $0 / 12$ anos & 1,00 & & & 1,00 & & & 1,00 & & \\
\hline $13 / 17$ anos & 1,50 & $(0,48-4,71)$ & & 0,32 & $(0,13-0,80)$ & & 1,05 & $(0,28-3,99)$ & \\
\hline 18 ou mais anos & 2,73 & $(0,88-8,48)$ & & 0,40 & $(0,16-1,00)$ & & 4,42 & $(1,25-15,65)$ & \\
\hline $\begin{array}{l}\text { Horas semanais } \\
\text { trabalhadas }\end{array}$ & & & 0,21 & & & 0,16 & & & 0,16 \\
\hline$\leq 35 \mathrm{~h}$ & 1,00 & & & 1,00 & & & 1,00 & & \\
\hline$\geq 36 \mathrm{~h}$ & 0,65 & $(0,33-1,27)$ & & 0,61 & $(0,31-1,20)$ & & 1,67 & $(0,82-3,40)$ & \\
\hline $\begin{array}{l}\text { Estrutura de } \\
\text { trabalho adequada }\end{array}$ & & & $0,004 \dagger$ & & & $0,01 \dagger$ & & & 0,49 \\
\hline Não & 1,00 & & & 1,00 & & & 1,00 & & \\
\hline Sim & 2,79 & $(1,40-5,59)$ & & 2,30 & $(1,17-4,53)$ & & 1,29 & $(0,63-2,62)$ & \\
\hline Apoio Social & & & $0,005 \dagger$ & & & 0,09 & & & 0,49 \\
\hline Baixo & 1,00 & & & 1,00 & & & 1,00 & & \\
\hline Alto & 2,74 & $(1,36-5,52)$ & & 1,82 & $(0,91-3,63)$ & & 1,29 & $(0,62-2,66)$ & \\
\hline
\end{tabular}

${ }^{*} \mathrm{p}$ de tendência linear. $\dagger \mathrm{p}$ de heterogeneidade.

dades, o que pode indicar maior controle sobre o trabalho.

As associações do apoio social com os quadrantes do MDC indicam que, quando os servidores podem contar com o apoio dos colegas e chefes, existe boa relação entre eles, as demandas psicológicas percebidas são baixas e o controle sobre o trabalho é alto (baixa exigência). No entanto, quando o ambiente é menos favorável e harmonioso, indicando baixo apoio social, as demandas psicológicas são elevadas e, se têm pouco controle sobre as situações laborais, configuram a alta exigência. A alta exigência é considerada a pior situação de estresse no trabalho, podendo gerar agravos à saúde ${ }^{9,12}$. Na cidade de Porto Alegre - RS, os sujeitos classificados nos quadrantes alta exigência/trabalho passivo, perceberam menor apoio social dos colegas e da chefia, comparados aos do grupo baixa exigência/trabalho ativo ${ }^{2}$. Outro estudo mostrou que os trabalhadores da equipe de enfermagem que interagiam menos com os colegas, característica de baixo apoio social, apresentaram maiores chances de ter alta exigência ${ }^{13}$.

$\mathrm{Na}$ regressão multinomial, as variáveis que permaneceram associadas foram: escolaridade, estrutura de trabalho e apoio social. A escolaridade que apresentou forte associação com o trabalho ativo, indicando que esses servidores são competentes em suas tarefas e possuem alto controle sobre o trabalho. Talvez por isso, sejam delegadas maiores demandas à parte desses indi- víduos. Ter boa estrutura de trabalho e alto apoio social dos colegas são fatores fundamentais na minimização do estresse laboral, uma vez que os sujeitos que classificaram esses itens como bons, estavam menos estressados, de acordo com a escala de estresse.

Algumas limitações do estudo devem ser relatadas. O número de perdas e recusas é algo que deve ser levado em consideração, visto que o mesmo pode, ter influenciado negativamente no encontro de possíveis associações, bem como na prevalência do desfecho. Provavelmente, indivíduos mais estressados foram aqueles que se recusaram a responder o questionário. Além disso, o estudo pode ter sido afetado por viés do trabalhador sadio, típico em estudos com trabalhadores que verificam desfechos em saúde. Outra limitação é referente à variável de estresse do Modelo Demanda-Controle que, por ser politômica, pode dificultar a compreensão dos resultados. Entretanto, o instrumento é o mais adequado para avaliar estresse laboral, via questionário, sendo utilizado em estudos de diferentes países, facilitando a comparação dos achados.

\section{Conclusões}

Boa parte dos servidores da UFPel está em condição de estresse laboral, indicado pelo quadrante de alta exigência. $\mathrm{O}$ estresse no trabalho mostrou- 
se associado à menor escolaridade, pior estrutura de trabalho e menor apoio social. Existem poucos estudos que apresentam associação entre estresse e escolaridade. Maior atenção à estrutura de trabalho e apoio social aos servidores deve ser foco de intervenção por parte dos gestores da universidade, no sentido de reduzir o nível de estresse e risco de adoecimento entre esses trabalhadores.

\section{Colaboradores}

SV Lopes participou de todas as etapas do estudo, incluindo: concepção do projeto, coleta de dados, análise e interpretação dos dados, redação e aprovação final da versão a ser publicada e foi responsável por todos os aspectos do trabalho na garantia de exatidão e integridade de qualquer parte da obra. MC Silva participou das seguintes etapas: concepção do projeto, análise e interpretação dos dados, revisão crítica relevante do conteúdo intelectual do texto e aprovação da versão a ser publicada.
Este é um dos poucos estudos a utilizar a Job Stress Scale em funcionários públicos no Rio Grande do Sul. Traçar o perfil de saúde dos servidores a partir de informações do ambiente de trabalho é importante para que sejam elaboradas estratégias de melhoria de condições laborais e políticas que beneficiem a saúde do servidor público.

\section{Agradecimentos}

Agradecemos a vice-reitora Denise Petrucci Gigante por autorizar o estudo nas unidades da UFPel e a todos os servidores que participaram do estudo. Esta pesquisa foi financiada pela Coordenação de Aperfeiçoamento de Pessoal de Nível Superior (Capes), por meio de bolsa de mestrado. 


\section{Referências}

1. Machida S. Stress prevention at work checkpoints. Geneva: International Labour Office; 2012.

2. Negeliskii C, Lautert L. Estresse laboral e capacidade para o trabalho de enfermeiros de um grupo hospitalar. Rev Latino Am Enfermagem 2011; 19(3):606-713.

3. Garbarino S, Cuomo G, Chiorri C, Magnavita N. Association of work-related stress with mental health problems in a special police force unit. BMJ 2013; 3(7):2791-2803.

4. Carder M, Turner S, McNamee R, Agius R. Work-related mental ill-health and 'stress' in the UK (2002-05). Occup Med 2009; 59(8):539-544.

5. Xu W, Zhao Y, Guo L, Guo Y, Gao W. Job stress and coronary heart disease: a case-control study using a chinese population. J Occup Health 2009; 51(2):107-113.

6. Health and Safety Executive (HSE). Stress and psychological disorders in great britain 2014 [periódico na Internet]. 2014 Out [acessado 2015 Abr 02]: 1(10) [cerca de 9 p.]. Disponível em: http://www.hse.gov.uk/statistics/causdis/stress/stress.pdf

7. Sadir MA, Bignotto MM, Lipp MEN. Stress e qualidade de vida: influência de algumas variáveis pessoais. Paideia 2010; 20(45):73-81.

8. Lopes CS, Faerstein E, Chor D. Eventos de vida produtores de estresse e transtornos mentais comuns: resultados do Estudo Pró-Saúde. Cad Saude Publica 2003; 19(6):1713-1720.

9. Araújo TM, Aquino E, Menezes G, Santos CO, Aguiar L. Aspectos psicossociais do trabalho e distúrbios psíquicos entre trabalhadoras de enfermagem. Rev Saude Publica 2003; 37(4):424-433.

10. Alves MGM, Chor D, Faerstein E, Werneck GL, Lopes CS. Estresse no trabalho e hipertensão arterial em mulheres no Estudo Pró-Saúde. Rev Saude Publica 2009; 43(5):883-886.

11. Karasek RA. Job demands, job decision latitude, and mental strain: inplications for job redesign. Admin Science Quarterly 1979; 24(2):285-308.

12. Alves MGM, Chor D, Faerstein E, Lopes CS, Werneckd GL. Versão resumida da "job stress scale": adaptação para o português. Rev Saude Publica 2004; 38(2):164171.

13. Urbanetto JS, Silva PC, Hoffmeister E, Negri BS, Costa BEP, Figueiredo CEP. Estresse no trabalho da enfermagem em hospital de pronto-socorro: análise usando a Job Stress Scale. Rev Lat Am Enfermagem 2011; 19(5):1122-1131

14. Minari MRT. Estresse em servidores públicos do instituto nacional de seguro social de Campo Gande-MS [dissertação]. Campo Grande: Universidade Católica Dom Bosco; 2007.

15. Armondes CCL, Vaz JT, Nozabieli AJL, Pachioni CAS, Fregonesi CEPT, Oikawa SM. Avaliação do estresse ocupacional em funcionários públicos. Rev Eletr Fisio FCT/UNESP 2009; 1(1):92-106.

16. Matsudo S, Araujo T, Matsudo V, Andrade D, Andrade E, Oliveira LC, Braggion G. Questionário internacional de atividade física (IPAQ): estudo de validade e reprodutibilidade no Brasil. Ativ Fis Saude 2001; 6(2):5-18.

17. Hallal PC, Gomez LF, Parra DC, Lobelo F, Mosquera J, Florindo AA, Reis RS, Pratt M, Sarmiento OL. Lessons learned after 10 years of IPAQ use in Brazil and Colombia. J Phys Act Health 2010; 7(2):259-264.
18. Office of Disease Prevention and Health Promotion. 2008 Physical activity guidelines for Americans. Washington: U.S. Department of Health and Human Services. [acessado 2015 Jun 07]: [cerca de 76 p.]. Disponível em: http://health.gov/paguidelines/guidelines/

19. World Health Organization (WHO). Obesity: preventing and managing the global epidemic. Geneva: WHO; 2000.

20. Hökerberg YH, Aguiar OB, Reichenheim M, Faerstein E, Valente JG, Fonseca MJ, Passos SR. Dimensional structure of the demand control support questionnaire: a Brazilian context. Int Arch Occup Environ Health 2010; 83(4):407-416.

21. Greco PBT, Magnago TSBS, Beck CLC, Urbanetto JS, Prochnow A. Estresse no trabalho em agentes dos centros de atendimento socioeducativo do Rio Grande do Sul. Rev Gau Enfermagem 2013; 34(1):94-103.

22. Brasil. Ministério da Saúde (MS). VIGITEL 2014: Vigilância de fatores de risco e proteção para doenças crônicas por inquérito telefônico. Secretaria de Vigilância em Saúde. Departamento de Vigilância de Doenças e Agravos não Transmissiviveis e Promoção da Saúde. Brasília: MS; 2015.

23. Linhares RS, Horta BL, Gigante DP, Dias-da-Costa JS, Olinto MTA. Distribuição de obesidade geral e abdominal em adultos de uma cidade no Sul do Brasil. Cad Saude Publica 2012; 28(3):438-448.

24. Silva KS, Nahas MV, Peres KG, Lopes AS. Fatores associados à atividade física, comportamento sedentário e participação na Educação Física em estudantes do Ensino Médio em Santa Catarina, Brasil. Cad Saude Publica 2009; 25(10):2187-2200.

25. Rocha SV, Pie ACS, Cardoso JP, Amorim CR, Carneiro LRV, Vilela ABA. Nível de atividade física entre funcionários de uma instituição de ensino superior da bahia. Ulbra Mov 2011; 2(1):16-29.

26. Costa VB, Soares NIS, Costa JES, Leone ID, Silva VF, Costa DCCO, Madeira FB, Cabral PUL. Nível de atividade física e qualidade de vida de funcionários públicos administrativos. Biomotriz 2014; 8(1):91-105.

27. Mark G, Smith AP. Occupational stress, job characteristics, coping, and the mental health of nurses. $\mathrm{Br} J$ Health Psychol 2012; 17(3):505-521.

28. Gonçalves E, Cosentino RC. Distress: qual a influência do exercício físico neste conceito. Rev Bras Cien Saude 2007; 3(12):50-54.

29. Johnson W, Krueger RF. The psychological benefits of vigorous exercise: a study of discordant MZ twin pairs. Twin Res Hum Genet 2007; 10(2):275-283

30. Proper K, van Mechelen W. Effectiveness and economic impact of worksite interventions to promote physical activity and healthy diet. Background paper prepared for the WHO/WEF Joint Event on Preventing Noncommunicable Diseases in the Workplace (Dalian/China, September 2007). Geneva: World Health Organization; 2008.

31. Nahas MV. Atividade Física, Saúde e Qualidade de Vida: Conceitos e Sugestões para um Estilo de Vida Ativo. Londrina: Midiograf; 2013.

32. Wemme KM, Rosvall M. Work related and non-work related stress in relation to low leisure time physical activity in a Swedish population. J Epidemiol Community Health 2005; 59(5):377-379. 
33. Lautizi M, Laschinger HKS, Ravazzolo S. Workplace empowerment, job satisfaction and job stress among Italian mental health nurses: An exploratory study. $J$ Nurs Manag 2009; 17(4):446-452.

34. Hamdan-Mansour AM, Al-Gamal E, Puskar K, Yacoub M, Marini A. Mental health nursing in Jordan: An investigation into experience, work stress and organizational support. Int J Ment Health Nurs 2011; 20(2):8694.

Artigo apresentado em 10/12/2015

Aprovado em 11/11/2016

Versão final apresentada em 13/11/2016 